\title{
A hybrid functional electrical stimulation for real-time estimation of joint torque and closed-loop control of muscle activation
}

\author{
Zhan Li (1,2), David Guiraud (1), David Andreu (1), Charles Fattal (3,4), Anthony \\ Gelis (3), Mitsuhiro Hayashibe (1) \\ (1) INRIA-LIRMM, University of Montpellier, Montpellier, France; (2) University of \\ Electronic Science and Technology of China, Chengdu, China; (3) PROPARA Rehabilitation \\ Center, Montpellier, France; (4) COS DIVIO, Dijon, France \\ This article is distributed under the terms of the Creative Commons Attribution Noncommercial License (CC BY-NC 4.0) which \\ permits any noncommercial use, distribution, and reproduction in any medium, provided the original author(s) and source are credited.
}

\begin{abstract}
As a neuroprosthetic technique, functional electrical stimulation (FES) can restore lost motor performance of impaired patients. Through delivering electrical pulses to target muscles, the joint movement can be eventually elicited. This work presents a real-time FES system which is able to deal with two neuroprosthetic missions: one is estimating FES-induced joint torque with evoked electromyograph (eEMG), and the other is artificially controlling muscle activation with such eEMG feedback. The clinical experiment results on spinal cord injured (SCI) patients and healthy subjects show promising performance of the proposed FES system.

Keywords: Functional electrical stimulation (FES); Torque prediction; Muscle activation control; Hybrid stimulation system
\end{abstract}

Eur J Transl Myol 26 (3): 193-196

Functional electrical stimulation (FES) is a neuroprosthetic technique aiming at assisting restoring motor functions of impaired patients who survive from spinal cord injury (SCI) or stroke, through delivery of electrical short pulses to target muscles. As a useful neuroprosthetic technique, FES has been widely applied among patients who suffer motor injury and neural disorder on motor restoring/retraining tasks, such as grasping or lifting objects, correcting drop foot during ambulation. Current FES systems are designed based on open-loop principle and have been widely used in clinical environment. Such open-loop based FES systems manipulate stimulation parameters (e.g., pulse width) manually without considering adaptive modeling and control between stimulation and muscle biofeedback and joint mechanical output. Such openloop systems lack feedback information to adjust model parameters and control configuration adaptively, which may degrade performances and limit application domains. To remedy disadvantages of open-loop FES systems, closed loop FES systems are to be developed for clinical environment ${ }^{1-3}$. Closed-loop FES systems possess better adaptivity in modeling and control but have not yet been widely applied for clinical uses. Evoked electromyography (eEMG) is considered as a direct image of electrical muscle activity under stimulation: it has been frequently used as biofeedback for control and estimation purposes in $\mathrm{FES}^{4-6}$. In this work, we present a hybrid real-time closed-loop FES system which both can control muscle activations and predict FES-induced torque based on eEMG. Such FES system was tested on able-bodied and SCI subjects, and promising joint torque prediction and muscle activation results demonstrate its feasibility and efficiency.

\section{Materials and Methods \\ System Architecture and Experiment Setup}

The hybrid FES system consisted of two functional parts. The first one controls muscle activation with eEMG feedback, and the second one estimates/predicts FES-induced joint torque with eEMG. The whole functionality of the system is shown on Fig. 1. Model predictive controller is designed in the control part and Kalman filter/recurrent neural network (RNN) is used in the torque estimation part ${ }^{6}$.

\section{Subjects}

The system tests were conducted on able-bodied subjects and SCI patients upon their consent with a protocol approved by Nimes Ethics Committee, France, 2013.

\section{System}

The system consists of a wireless stimulator $^{7}$ (emanating from a transfer of our technology to Vivaltis 


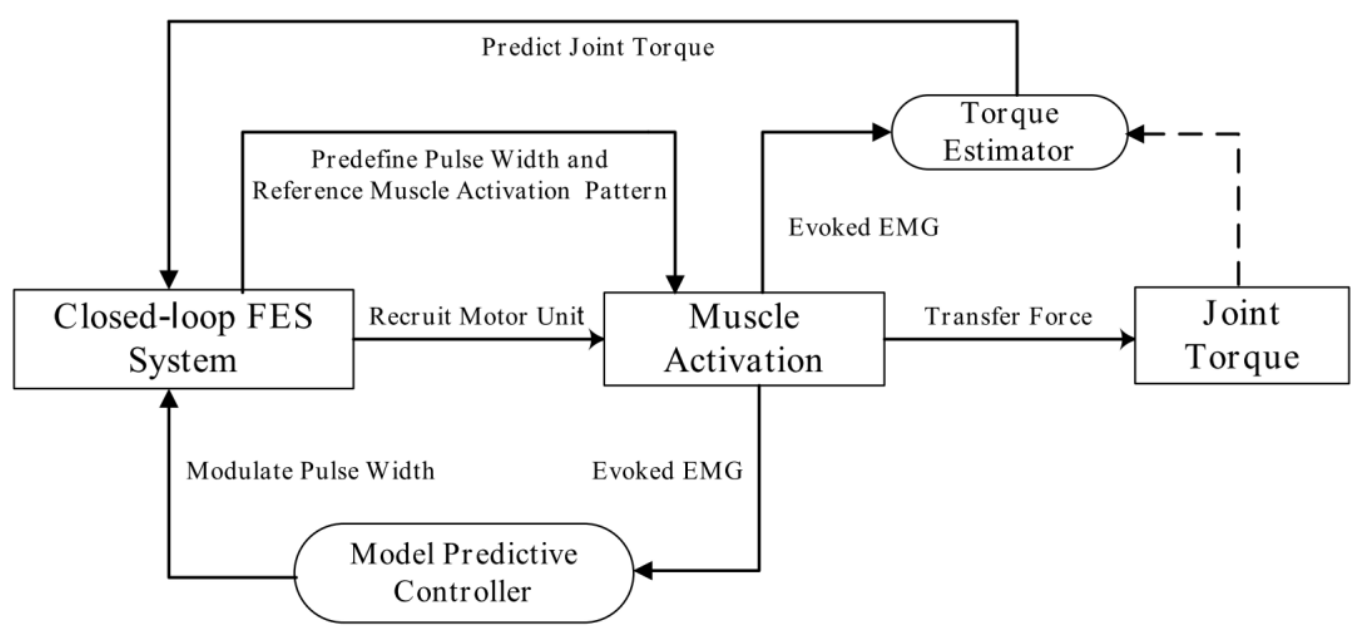

Fig 1. The architecture diagram of the hybrid FES system. The system contains two functionality parts: one is to control muscle activations with eEMG feedback and the other is to predict FES-induced torque based on eEMG.

Inc., Montpellier, France), eEMG (MP100, Biopac Systems Inc., Santa Barbara, CA, USA), torque acquisition devices (Biodex 3, Shirley Corp., NY, USA), and a laptop computer with the MATLAB (version 2012a) interface for remote control of wireless stimulator and real-time data acquisition/ processing. The wireless stimulator is a regulated current dualchannel stimulator. The main features of the wireless stimulator are as follows: maximal current is $100 \mathrm{~mA}$ with $0 \mathrm{~mA}$ to $1 \mathrm{~mA}$ step on a maximum load of $1 \mathrm{~kW}$, stimulation frequency ranges from $1 \mathrm{~Hz}$ to $1 \mathrm{kHz}$, onset pulse-width is $50 \mathrm{~ms}$ with $1 \mathrm{~ms}$ step, and electrical polarity can be configured according as required. Pulse width and Intensity are dynamically and remotely adjustable. The maximum pulse width for the experiment is limited to $450 \mathrm{~ms}$, the stimulation intensity is chosen between $25 \mathrm{~mA}$ and $35 \mathrm{~mA}$, and the stimulation frequency is chosen between $30 \mathrm{~Hz}-40 \mathrm{~Hz}$ to ensure a good fusion of muscle's contraction.

For eEMG acquisition, the bipolar $\mathrm{AgCl}$ EMG electrodes were positioned over the muscle belly in the direction of muscle fiber with $20 \mathrm{~mm}$ interelectrode spacing. The reference electrode was placed on the patella of contra lateral leg. Electrical current pulses were delivered to the Tibialis (TA) or Medial Gastrocnemius (MG) muscle group with surface electrodes placed. The raw eEMG signals are acquired at a sampling frequency fs $=4096 \mathrm{~Hz}$ through Biopac 100 system in order to capture the full range samplings of M-wave. The real-time identification and control are performed between 2 stimulation pulses so that the update and control frequency is the same as the stimulation frequency. The computation window for the eEMGis thus of the length of the period between 2 pulses i.e. around Nwin = fs/ fstim) where fs and fstim denote the eEMG sampling frequency and stimulation frequency respectively. Stimulation artifact suppression and mean-absolute operations using eEMG signals are performed.

The blanking template operation is used to suppress the stimulation artifacts $^{8}$, and such blanking operation is performed in every Nwin samplings of eEMG at a frequency fstim. The mean absolute values (MAV) of the Nwin samplings eEMG are computed at a frequency fstim, and then the muscle activations under every stimulation loop are obtained through normalization of

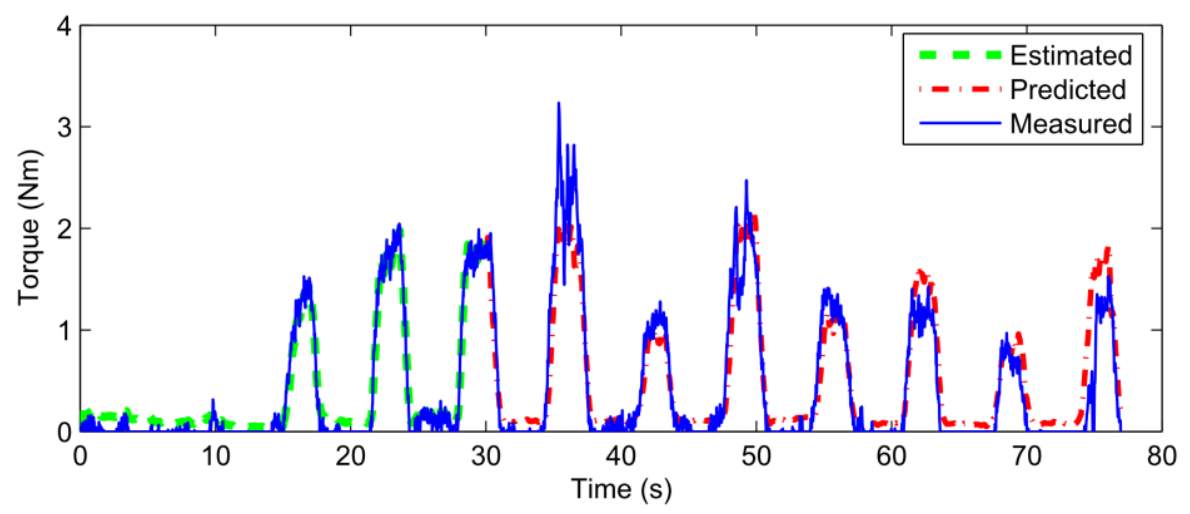

Fig 2. Prediction of FES-induced ankle joint torque with eEMG for a single SCI patient. 

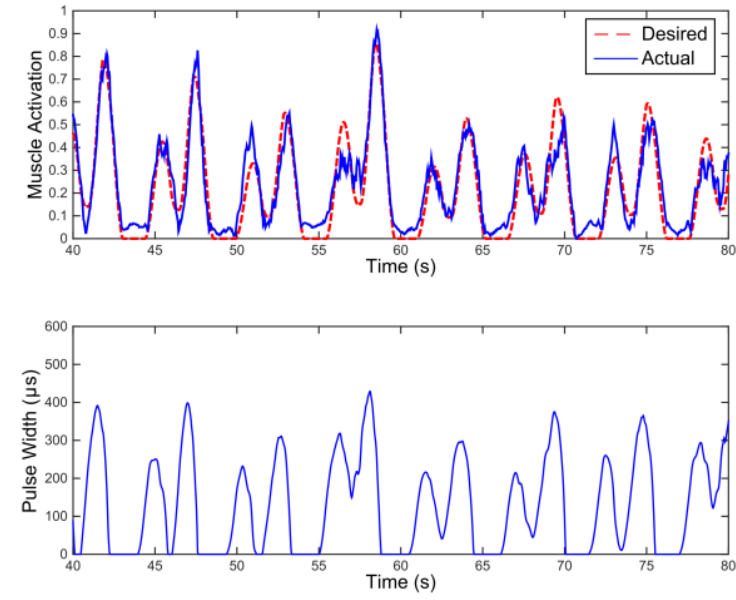

(a) "Dual sinusoidal" muscle activation pattern
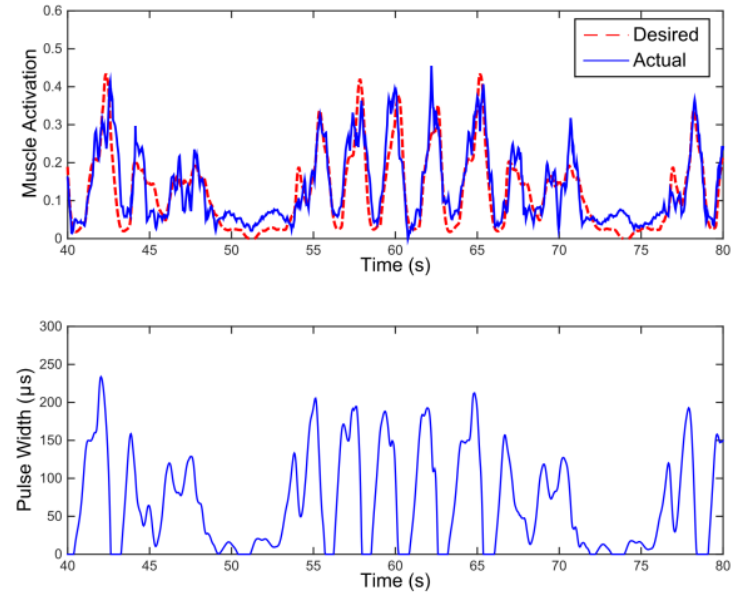

(b) "Walking cycle" muscle activation pattern

Fig 3. (a) "Dual sinusoidal" muscle activation pattern; (b) "Walking cycle" muscle activation pattern. Real-time control results on one SCI patient. Upper of subfigure: real-time control performance of muscle activation with desired muscle activation patterns (red dash line is desired muscle activation trajectory and blue solid line is the measured muscle activation under the muscle activation control by FES). Lower of subfigure: the corresponding computed stimulation pulse width, which was systematically generated by the predictive FES controller.

the maximum MAV of eEMG against their maximum. For joint torque estimation computations, the subject needed to be seated on the chair with the ankle at 90 degrees, while the joint center was aligned with the axis of a calibrated dynamometer. The shank was adjusted with the knee joint at 40 degrees. The foot was strapped to the pedal to transmit ankle torque to the dynamometer and to allow the optimal recording of isometric/non-isometric ankle torque. Raw eEMG of TA or MG muscle group and ankle joint torque were recorded, amplified (gain 1000) and sampled at a frequency fsamp $=4096 \mathrm{~Hz}$ through the Biopac amplifier and a 16-bit A/D national instrument card.

\section{Results}

\section{FES-induced torque prediction with eEMG}

The stimulation frequency fstim was set between 30 and $40 \mathrm{~Hz}$ leading to the loop execution time between $25 \mathrm{~ms}$ and $33 \mathrm{~ms}$. The suitable stimulation intensities were found to be from $20 \mathrm{~mA}$ to $50 \mathrm{~mA}$ specific to each patient. Then the stimulation intensity is fixed and PW modulation is performed to have different levels of muscle contractions to induce regular joint movement. The test session included 2 phases: identification and prediction phases. Each sequence contained trapezoidal trains consisting of $2 \mathrm{~s}$ stimulation $(0.5 \mathrm{~s}$ ramp up, $1 \mathrm{~s}$ plateau and $0.5 \mathrm{~s}$ ramp-down) and $2 \mathrm{~s}$ rest. During identification phase that lasts between 33 and 40s, the plateau stimulation PW of each trapezoidal train was increased gradually with step size of from $40 \%$ to $100 \%$ of the maximum PW. After identification, the plateau stimulation PW was randomly determined within 50\% to $100 \%$ of maximum PW in the prediction phase. The stimulation artifacts in the raw eEMG were removed by blanking with a window size at $10 \mathrm{~ms}$. Both the stimulus-activation model and activation-torque model are identified during the same identification phase. Fig. 2 shows the ankle joint torque prediction results based on RNN which was proposed ${ }^{6}$ for one SCI patient. Observed from the figure, we can see the joint torque prediction seems promising on the SCI patient, with root mean square error (RMSE) being $0.24 \mathrm{Nm}$ and variance accounted for (VAF) being $78.75 \%$ respectively.

\section{Muscle Activation Control with eEMG}

Afterwards, the model predictive controller is computing the PW level with PW-NMAV model updated at every stimulation loop and send the PW value to the wireless stimulator to perform the tracking control. Four different reference patterns of muscle activation under FES were tested and part of these results are presented: Every control loop is executed within $20 \mathrm{~ms}$, which does not exceed the maximum allowable time period for every stimulation loop $\mathrm{k}$. In this paper, the desired muscle activation for the realtime control by FES is generated artificially following two patterns: 1) One kind of natural patterns with two continuous sinusoidal contraction ${ }^{9}$ for drop foot correction; 2) Actual muscle contraction pattern measured by EMG envelope from the able-bodied subject during a whole cycle of actual walking test. Fig. 3 shows the control results on one SCI patient, we could see from this figure that the muscle activation controlled by the FES controller can track the desired reference quite well. 


\section{A hybrid FES for real-time torque estimation and muscle activation control}

Eur J Transl Myol 26 (3): 193-196

\section{Discussion}

This paper presents a hybrid FES system which can control muscle activation and predict joint torque with eEMG. Clinical experiment results on SCI subjects verify its feasibility and efficiency. Future work could be extended to applying such FES system on estimation/control issues of multiple muscles/joints with muscle/joint synergy considered ${ }^{10}$, and comparison of M-wave during dynamic contractions and during isometric contractions is also worth investigating.

\section{Authors' Contributions}

Zhan Li: Experiment preparation, data acquisition and processing, publication writing; David Guiraud: Experiment preparation, supervision of data processing and publication drafting, publication review; David Andreu: FES system implementation and technology support, publication review; Charles Fattal: Experiment preparation, medical support; Anthony Gelis: Experiment preparation, medical support; Mitsuhiro Hayashibe: Experiment preparation, data acquisition, supervision of data processing, publication review.

\section{Acknowledgement}

The authors wish to thank all PROPARA therapists and the patients. This paper was prepared for the IFESS Conference 2016.

\section{Conflict of interest}

The authors declare no conflict of interest.

\section{Corresponding author}

Mitsuhiro Hayashibe, LIRMM - Université de Montpellier, 860 Rue de St Priest, 34095 Montpellier cedex 5 - France

E-mail: hayashibe@lirmm.fr

\section{E-mails of coAuthors}

Zhan Li, zhan.li@uestc.edu.cn

David Guiraud, guiraud@lirmm.fr

David Andreu, andreu@lirmm.fr

Charles Fattal, cfattal@cos-asso.org

Anthony Gelis, A.GELIS@propara.fr

\section{References}

1. Li Z, Hayashibe M, Andreu D, Guiraud D. Real-time closed-loop fes control of muscle activation with evoked emg feedback, in Neural Engineering (NER), 2015 7th International IEEE/EMBS Conference 2015;623-626.

2. Jezernik S, Wassink R, Keller T. Sliding mode closed-loop control of fes controlling the shank movement, Biomedical Engineering, IEEE Transactions 2004; 51:263-272.

3. Azevedo C, Sijobert B, Froger J, Fattal C, Preliminary developments towards closed-loop fesassistance of posture and gait, IFAC PapersOnLine 2015;48:333 - 337.

4. Chen J, Yu NY. The validity of stimulus-evoked emg for studying muscle fatigue characteristics of paraplegic subjects during dynamic cycling movement, Rehabilitation Engineering, IEEE Transactions 1997;5:170-178.

5. Erfanian A, Chizeck H, Hashemi R. Using evoked emg as a synthetic force sensor of isometric electrically stimulated muscle. Biomedical Engineering, IEEE Transactions 1998;45:188-202.

6. Li Z, Hayashibe M, Fattal C, Guiraud D. Muscle fatigue tracking with evoked emg via recurrent neural network: Toward personalized neuroprosthetics. Computational Intelligence Magazine IEEE 2014;9: 38-46.

7. Toussaint M, Andreu D, Fraisse P, Guiraud D. Wireless distributed architecture for therapeutic functional electrical stimulation: a technology to design network-based muscle control. Engineering in Medicine and Biology Society (EMBC), 2010 Annual International Conference of the IEEE 2010;6218-6221.

8. Frigo C, Ferrarin M, Frasson W, Pavan E, Thorsen R. Emg signals detection and processing for on-line control of functional electrical stimulation," J Electromyogr Kinesiol 2000;10:351-360.

9. O'Keeffe DT, Donnelly AE, Lyons GM. The development of a potential optimized stimulation intensity envelope for drop foot applications, Neural Systems and Rehabilitation Engineering, IEEE Transactions 2003;11:249-56.

10 Li Z, Guiraud D, Hayashibe M. Inverse estimation of multiple muscle activations from joint moment with muscle synergy extraction. IEEE J Biomed Health Inform. 2015;19:64-73. doi: 10.1109/JBHI.2014. 2342274. Epub 2014 Jul 23. 